\title{
$0=$ \\ An educational initiative to improve the team-based care of patients with idiopathic pulmonary fibrosis
}

To the Editor:

The management of idiopathic pulmonary fibrosis (IPF) is complex, as is the process of implementing and assessing a set of quality indicators representing best care practices in IPF by an interstitial lung disease (ILD) programme $[1,2]$. To date, there is limited literature documenting the importance of IPF interventions to improve coordination of care, patient engagement in health literacy and education, and understanding what is important to patients [3-8]. In 2015, National Jewish Health (NJH) engaged our ILD division healthcare professionals (10 physicians, 4 nurses, 2 medical assistants, 1 physician assistant) and our professional education and biostatistics teams to design and implement a project aimed at measuring key quality indicators and how they may impact clinical practice and IPF patient perception of care.

Using the Plan-Do-Study-Act (PDSA) stepwise approach, the ILD team reviewed the current practices and processes related to caring for patients with IPF and identified eight metrics to be addressed in the study. The metrics were followed prospectively and measured quarterly between January 15, 2016 and January 15, 2017 ( $\mathrm{n}=587$ IPF patients) by physicians individually and as an ILD group aggregate, and then compared to the calendar year 2014 as a baseline (n=276 IPF patients). Metrics were selected and developed through a blended approach of baseline electronic medical record (EMR) audits, interviews with members of the interdisciplinary ILD team and a peer-reviewed literature review. They included discussing, ordering and recording immunizations, 6-minute walk tests (6-MWTs), nocturnal oximetry and oxygen titration, as well as executing a weight-loss intervention, placing referrals to resources such as education classes and pulmonary rehabilitation and discussing reflux with patients [1, 9-14].

Based on this formative work, we employed the following strategies during the first 3 months of the initiative: 1) Baseline EMR reports were distributed to individual providers to assess their own performance on the metrics listed, 2) Providers were asked to complete a self-reflection survey in response to their feedback reports on key quality performance measures, and 3) The ILD nurses were trained to navigate the patient through participation in systems-based resources (i.e. pulmonary rehabilitation, vaccination documentation and orders by the clinician etc.), work closely to track provider performance and patient data, and assist the healthcare team in providing patient education.

Every 4 months, throughout the implementation of the second PDSA cycle, education and training was disseminated to the ILD team. The education addressed the gaps identified in the needs assessment, including new processes for documentation and tracking, non-pharmacologic therapies for IPF, and patient education strategies while multidisciplinary team training included role-play demonstrations and EMR documentation training.

We developed a patient education activation card to use in clinic with patients diagnosed with IPF (figure 1). Of critical importance was the process by which the card was to be introduced and discussed with patients. Four ILD division nurses were targeted to lead a specific patient meeting following the

@ERSpublications

A successful initiative to improve best care practice in IPF supported by electronic medical record changes http://ow.ly/ORxi30hBEmy

Cite this article as: Fernández Pérez ER, Zelarney P, Thomas S, et al. An educational initiative to improve the team-based care of patients with idiopathic pulmonary fibrosis. ERJ Open Res 2018; 4: 00093-2017 [https://doi.org/10.1183/23120541.00093-2017]. 
physician visit to review the card, answer questions and direct patients to additional resources as requested.

In addition to the card, several other interventions were employed during the initiative, including two order sets developed specifically for IPF patient visits. One order set consolidated the list of key quality indicators identified by the team, while another showed all education classes available and appropriate to IPF patients at NJH, such as Managing Your Lung Disease and On The Go With Oxygen. ILD division physicians were also provided with a dictated note upon completion of each IPF patient visit, which served as a reminder to document all appropriate conversations, recommendations and actions taken with the patient regarding the eight metrics.

IPF patients who received the education activation card, as well as those who did not, and ILD division providers were surveyed at initiative conclusion and focus groups were conducted among patients and ILD division nurses by HealthCare Research Inc (Denver, CO, USA). Percentages were used to describe categorical variables. Data were entered into JMP statistical software (Version 13.1, SAS Institute Inc) with metrics scored as a binary variable. Individual and group metrics were calculated and compared as a sum of completed quality metrics. The primary outcome measure was the proportion of metrics per chart (at least one per patient) at each quarterly visit in compliance with the documented eight metrics and the nurse-driven education card intervention. The reliability and validity of the database curator process used to abstract and measure all metric variables from the EMR were reviewed weekly and analysed for discrepancies by the study authors. The National Jewish Health Institutional Review Board approved the study.

Aggregate provider metrics improved over time in all areas measured. Overall, the average relative percentage change in documentation and behaviours related to each of the eight metrics increased $141 \%$ from baseline to initiative conclusion (27\% for calendar year 2014 to $65 \%$ for Q4 of 2016). Focus groups aided our understanding of how the new approaches were received by healthcare professionals and by patients. Patient participants were recruited by phone using a list of nearly 100 IPF patients. Most of the patients on the list had been seen at the NJH ILD clinic for the first time within the previous 6 months. Ten patient participants were recruited for the focus group discussion. Additionally, both IPF patients and ILD division physicians were surveyed at initiative conclusion. While provider metrics improved, patient surveys $(n=50)$ indicated that many patients, often the majority, do not recall their provider discussing important issues of IPF disease expectations and management. Patient focus group analysis indicated similar conclusions. When we showed group participants the patient education activation card developed in this initiative, it was regarded as an important part of a conversation with a clinician at the time of

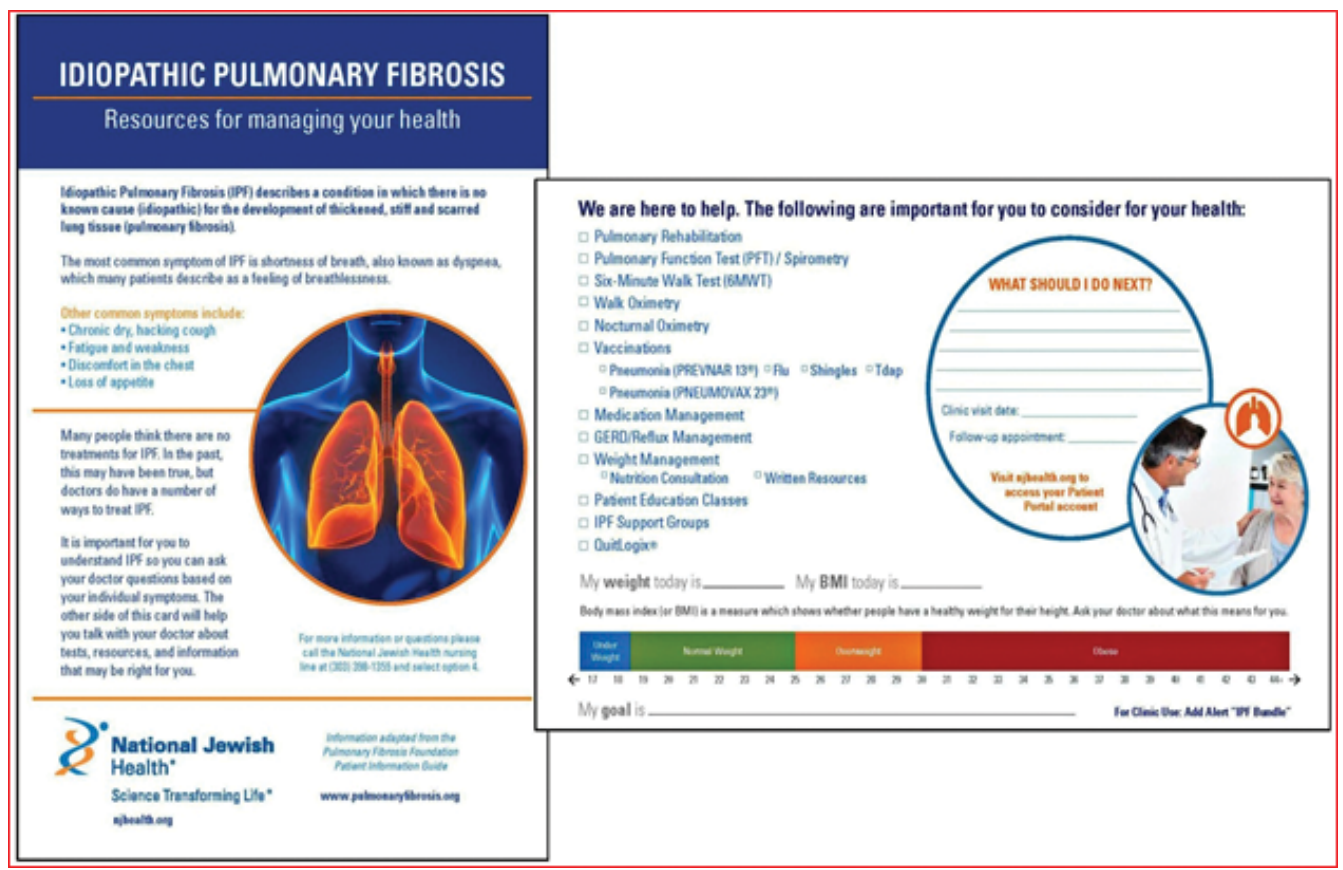

FIGURE 1 A patient education activation card. This card was developed for use by health care providers at National Jewish Health (NJH) to assess care of their patients with idiopathic pulmonary fibrosis (IPF). Metrics for IPF care were selected using clinical recommendations and developed in collaboration with a multidisciplinary clinic team. 
diagnosis. Similar too was the nurse focus group feedback: the card was critical if a process flow was put in place in clinic, because it allowed them to approach or continue important conversations at the appropriate time for the patient.

ILD division providers were surveyed at initiative conclusion to provide feedback on specific elements of the programme. Of these providers, $71 \%$ stated that through the programme they recognised the benefit of addressing topics such as weight management and gastroesophageal reflux disease (GERD) with their IPF patients and $51 \%$ noted that the programme motivated them to talk about and/or refer IPF patients to organisational support resources such as education classes or support groups. All providers (100\%) stated that the programme motivated them to intentionally involve the ILD nurses more in the IPF patient visit and to talk about and/or refer to organisational support resources. When asked about the perceived sustainability of these practice changes, $43 \%$ felt they were sustainable with the rest (57\%) indicating that they were somewhat sustainable. Physicians and nurses indicated more time as the most important factor in sustainably incorporating these best practices.

There is variation in IPF management in the outpatient setting. Adherence to IPF best practice interventions provides a systematic standardised approach to IPF management. Establishing disease-management interventions for IPF care may result in the reduction of symptom burden for patients with IPF and their care partners and potentially influence IPF prognosis [7, 14]. We have demonstrated that it is possible to improve performance on measures of IPF care through an intensive educational, practice improvement initiative. Furthermore, we have identified a need for improved documentation of quality indicators and have evaluated the effect of an IPF patient education activation card in promoting a patient-centered IPF team-based approach. The main findings from this study were as follows: 1) Streamlining processes for the entire team aids in improving implementation of best practices overall and multidisciplinary team collaboration, 2) Time to deliver patient education is limited, thus engaging the team can both address the barrier and empower clinical team members, and 3) Providing take-away resources (e.g. the education card) and incorporating it during conversations at various times, rather than just upon the initial ILD visit, is vital for improving patient communication and education.

This study has limitations. First, it took place at a single tertiary academic centre and success required the training of the ILD physician and office staff, as well as information technology system availability, use and training to enable the ILD physician to track and monitor IPF patients (with respect to core measures reporting through monthly feedback, benchmarking and the generation of reminders for physicians designed to support them in reaching goals). As such, external validation studies and application of the interventions at smaller programmes with fewer resources is needed. Secondly, although the multifaceted interventions implemented in the study (e.g. monthly physician reminders, audit and feedback from benchmark reports) are more likely to be effective in changing clinical practice behaviour compared with single interventions, it is unclear if the implemented methods are enduring and to what extent optimising management of IPF through effective healthcare provider-patient communication and education improves, if at all, the patient quality of life. Thirdly, the feedback provided by patients was not adjusted by their level of education. However, this is unlikely to have a tangible effect on the study results given the homogenous results of the patient survey demonstrating improvement in measures of the patient-centered care experience (such as being better informed about IPF and more satisfied with the care they received). Finally, specific patient-reported outcomes, such as measures of depression and function were not evaluated to further assess the effectiveness of the programme.

In conclusion, the initiative was successful in improving documentation of best care practices with IPF and adjustments to the EMR to capture these elements supported sustainability. The initiative identified a need to help our IPF patients understand and manage their disease and highlighted the importance of harnessing patient education time by addressing clinic workflow productivity. More prospective research is required in this field to identify IPF patient and caregiver-centered needs in order to build better interventions and optimise patient educational experience and provider-patient communication.

Evans R. Fernández Pérez ${ }^{1}$, Pearlanne Zelarney ${ }^{2}$, Shirley Thomas ${ }^{3}$, Deborah A. Shoop ${ }^{3}$, Jessica L. Munson ${ }^{3}$, Kelsey D. Johnson ${ }^{3}$, Matthew E. Stern ${ }^{4}$ and Sarah Meadows $\oplus^{4}$

${ }^{1}$ Division of Pulmonary, Critical Care and Sleep Medicine, National Jewish Health, Denver, CO, USA. ${ }^{2}$ Dept of Research Informatics, National Jewish Health, Denver, CO, USA. ${ }^{3}$ Dept of Nursing, National Jewish Health, Denver, CO, USA. ${ }^{4}$ Dept of Professional Education, National Jewish Health, Denver, CO, USA.

Correspondence: E.R. Fernández Pérez, Division of Pulmonary, Critical Care and Sleep Medicine, Interstitial Lung Disease Program and Autoimmune Lung Center, National Jewish Health, Southside Building, Office \#G12, 1400 Jackson Street, Denver, CO 80206, USA. E-mail: Fernandezevans@njhealth.org 
Received: July 272017 | Accepted after revision: Dec 202017

Support statement: This study was support by Genentech Inc. Funding information for this article has been deposited with the Crossref Funder Registry.

Conflict of Interest: E.R. Fernández Pérez has received consulting fees from Genentech and Boehringer Ingelheim and speaking fees related to IPF from Genentech and Boehringer Ingelheim.

Acknowledgements: The authors are grateful for the support provided by the interstitial lung disease team at National Jewish Health.

\section{References}

1 Raghu G, Collard HR, Egan JJ, et al. An official ATS/ERS/JRS/ALAT statement: idiopathic pulmonary fibrosis: evidence-based guidelines for diagnosis and management. Am J Respir Crit Care Med 2011; 183: 788-824.

2 Thickett DR, Kendall C, Spencer LG, et al. Improving care for patients with idiopathic pulmonary fibrosis (IPF) in the UK: a round table discussion. Thorax 2014; 69: 1136-1140.

3 Russell AM, Sprangers MA, Wibberley S, et al. The need for patient-centred clinical research in idiopathic pulmonary fibrosis. BMC Med 2015; 13: 240.

4 Schoenheit G, Becattelli I, Cohen AH. Living with idiopathic pulmonary fibrosis: an in-depth qualitative survey of European patients. Chron Respir Dis 2011; 8: 225-231.

5 Collard HR, Bradford WZ, Cottin V, et al. A new era in idiopathic pulmonary fibrosis: considerations for future clinical trials. Eur Respir J 2015; 46: 243-249.

6 Wuyts WA, Peccatori FA, Russell AM. Patient-centred management in idiopathic pulmonary fibrosis: similar themes in three communication models. Eur Respir Rev 2014; 23: 231-238.

7 Lindell KO, Olshansky E, Song MK, et al. Impact of a disease-management program on symptom burden and health-related quality of life in patients with idiopathic pulmonary fibrosis and their care partners. Heart Lung 2010; 39: 304-313.

8 Cottin V, Bourdin A, Crestani B, et al. Healthcare pathway and patients' expectations in pulmonary fibrosis. ERJ Open Research 2017; 3: 1-3.

9 Raghu G, Rochwerg B, Zhang Y, et al. An official ATS/ERS/JRS/ALAT clinical practice guideline: treatment of idiopathic pulmonary fibrosis. An update of the 2011 clinical practice guideline. Am J Respir Crit Care Med 2015; 192: e3-e19.

10 Johannson KA, Strambu I, Ravaglia C, et al. Antacid therapy in idiopathic pulmonary fibrosis: more questions than answers? Lancet Respir Med 2017; 5: 591-598.

11 Chandrashekaran S, Keller CA, Kremers WK, et al. Weight loss prior to lung transplantation is associated with improved survival. J Heart Lung Transplant 2015; 34: 651-657.

12 National Clinical Guideline Centre. Diagnosis and management of suspected idiopathic pulmonary fibrosis London, National Clinical Guideline Centre, 2013. www.ncbi.nlm.nih.gov/pubmedhealth/PMH0068972/pdf/ PubMedHealth_PMH0068972.pdf Date last accessed: January 11, 2018.

13 Clark M, Cooper B, Singh S, et al. A survey of nocturnal hypoxaemia and health related quality of life in patients with cryptogenic fibrosing alveolitis. Thorax 2001; 56: 482-486.

14 Kulkarni T, Willoughby J, Acosta Lara Mdel P, et al. A bundled care approach to patients with idiopathic pulmonary fibrosis improves transplant-free survival. Respir Med 2016; 115: 33-38. 